\title{
Subjective Uncertainty Over Behavior Strategies: A Correction
}

\section{Citation}

Dekel, Eddie, Drew Fudenberg, and David K. Levine. 2002. Subjective uncertainty over behavior strategies: A correction. Journal of Economic Theory 104, no. 2: 473-478.

\section{Published Version}

http://dx.doi.org/10.1006/jeth.2001.2866

\section{Permanent link}

http://nrs.harvard.edu/urn-3:HUL.InstRepos:3200611

\section{Terms of Use}

This article was downloaded from Harvard University's DASH repository, and is made available under the terms and conditions applicable to Other Posted Material, as set forth at http:// nrs.harvard.edu/urn-3:HUL.InstRepos:dash.current.terms-of-use\#LAA

\section{Share Your Story}

The Harvard community has made this article openly available.

Please share how this access benefits you. Submit a story.

\section{Accessibility}




\section{Subjective Uncertainty over Behavior Strategies: A Correction *}

Running Title: Uncertainty over Behavior Strategies

Eddie Dekel, Drew Fudenberg, and David K. Levine

November 2000

Revised May 2001

Eddie Dekel: Departments of Economics, Northwestern and Tel Aviv Universities, dekel@ northwestern.edu

Drew Fudenberg: Department of Economics, Harvard University, dfudenberg@ harvard.edu

David K. Levine: Department of Economics, ULCA, dlevine@ucla.edu

Send manuscript correspondence to Drew Fudenberg, Department of Economics, Harvard University, Cambridge MA 02138; phone (617) 496-5895, fax (617) 495 -4341.

* This work is supported by the National Science Foundation under Grants 99-86170, 97-30181, and 9730493. 


\begin{abstract}
In order to model the subjective uncertainty of a player over the behavior strategies of an opponent, one must consider the player $i$ 's beliefs about the opponents' play at information sets that player $i$ thinks have probability 0 . This erratum uses "trembles" to provide a definition of the convex hull of a set of behavior strategies. This corrects a definition we gave in [5], which led two of the solution concepts we defined there to not have the properties we intended.
\end{abstract}




\section{Introduction}

Rationalizability and related concepts are defined and characterized in terms of sets of strategies for a player that other players think he might use. In the strategic form each player's beliefs about the play of an opponent are given by a probability measure over this set, and each such measure maps to a point in the convex hull of the set of possible strategies, as in Bernheim [3] and Pearce [9]. Consequently, we can take this convex hull as a model of what players might think about other players. In extensiveform models that use behavior strategies, the correct way to model beliefs and map them to strategies is less transparent. Geir Asheim has pointed out to us that in Dekel, Fudenberg and Levine [5] we give an incorrect definition of convex combinations of behavior strategies. As a result two of the concepts that we defined (sequential rationalizability and sequentially rationalizable self-confirming equilibrium) do not have the properties that the paper implies and intended. This erratum uses "trembles" to provide a definition of mixtures that, when embedded in our definitions of sequential rationalizability and sequentially rationalizable self-confirming equilibrium, makes them function in the way we intended. ${ }^{1}$ In particular, with the corrected definition it will be the case that when a player thinks that only a single behavior strategy is consistent with rational play by his opponent, his beliefs about that opponent correspond to that unique behavior strategy. Consequently, sequential rationalizability implies backwards induction in finite games of perfect information with generic payoffs.

Instead of using trembles, Asheim and Perea [1] use lexicographic probability systems (extending Blume, Brandenburger and Dekel [4]) to model players' beliefs in extensive-form games; among other things they use these systems to provide a correct definition of sequential rationalizability for two-player games. Battigalli [3] models beliefs in extensive-form games using Myerson's [8] conditional probability systems, to which lexicographic probability systems are closely related. We prefer to use trembles instead because we already used them in another part of our 1999 paper, and because for

\footnotetext{
${ }^{1}$ As we explain below, the error does not matter for the solution concept of rationalizable self-confirming equilibrium, which was the primary focus of that paper.
} 
our purposes it is not necessary to track the relative likelihoods of various zeroprobability events. $^{2}$

\section{Preliminaries}

To save space we will assume that the reader is familiar with most of the notation and terminology of Dekel, Fudenberg, and Levine [5], and so we will only restate a few of the most relevant definitions. An assessment $a_{i}$ for player $i$ is a probability distribution over nodes at each of his information sets. A belief for player $i$ is a pair $b_{i} \equiv\left(a_{i}, \pi_{-i}^{i}\right)$, consisting of $i$ 's assessment over nodes $a_{i}$, and $i$ 's expectations of opponents' strategies $\pi_{-i}^{i}$ $=\left(\pi_{j}^{i}\right)_{j \neq i .}^{3}$ In that paper we defined belief closed as follows:

Definition 2.2: A belief model $V$ is belief closed if for every $\left(\pi_{i},\left(a_{i}, \pi_{-i}^{i}\right)\right) \in V_{i}, \pi_{j}^{i}$ arises from a mixture over strategies in the set $\left\{\pi^{\prime}{ }_{j} \mid\left(\pi^{\prime}{ }_{j}, b_{j}\right) \in V_{j}\right.$ for some belief $\left.b_{j}\right\}$.

This definition is silent on what it means to say that a behavior strategy "arises from a mixture" over other behavior strategies; the paper elaborates in footnote 11, which says:

"A behavior strategy $\pi_{j}$ is generated by a mixture $(\alpha, 1-\alpha)$ over $\pi_{j}$ ' and $\pi_{j}{ }^{\prime}$ if for every $\pi_{-j}$, the distribution over terminal nodes induced by $\left(\pi_{j}, \pi_{-j}\right)$ equals the $(\alpha, 1-\alpha)$ mixture over the distributions induced by $\left(\pi_{j}, \pi_{-j}\right)$ and $\left(\pi_{j}, \pi_{-j}\right)$ respectively."

This "clarification" is incorrect. The problems arise in defining the behavior of $\pi_{j}^{i}$ at information sets for $j$ that are not reachable under any of the strategies in $V_{j} \cdot{ }^{4}$ Such information sets are irrelevant for concepts that place no restrictions on play at

\footnotetext{
${ }^{2}$ Contemporaneously with this paper, Asheim and Perea developed a trembles-based alternative to their use of lexicographic probability systems.

${ }^{3}$ The assumption that player $i$ 's expectations about an opponents' play corresponds to a strategy profile incorporates the implicit restriction that opponents randomize independently. Note that what we call an "assessment" is what Kreps and Wilson [8] call a "system of beliefs for player $i$," and that our "belief" is similar to what they call an "assessment."

${ }^{4}$ That is, an information set is unreachable under $\pi_{j}$ if there is no profile $\pi_{-j}$ for $j$ 's opponents such that the information set is reached with positive probability.
} 
information sets that the strategy precludes, but the mistake is important for concepts such as our sequential rationalizability that impose restrictions on play at all information sets.

For example consider the game in Figure 1:

<put Figure 1 here>

The backwards induction profile is $\pi_{1}=$ (in, up) and $\pi_{2}=$ (across); the profile ((out, down), (down)) is an imperfect Nash equilibrium. Only up is sequentially rational at player 1's second information set, so the set of sequentially rational behavior strategies for player 1 must contain only strategies that play up at this information set. Consider the sets $V_{1}=(($ out, up $)$, down $), V_{2}=($ down,$($ out, down $))$. Since the strategy (out, down) is equivalent in the strategic form to (out, up), it "arises as a mixture over" the set of player 1's strategies in $V_{1}$. Consequently, this pair is sequentially rational and belief closed when "arises from" is defined as in footnote 11. In particular, sequential rationality and belief-closed with the original definition does not imply backwards induction.

\section{The Extensive-Form Convex Hull}

We therefore propose the following definition of the "convex hull" of behavior strategies, which corrects and builds from our previous definition by using "trembles" to make sure that every information set of player $i$ is reachable. ${ }^{5} \quad$ When working with strictly positive behavior strategies, there are no unreached information sets. In this case the definition of "generated by a mixture" from our previous paper is adequate.

Definition: Strategy $\pi_{i}$ is in the extensive-form convex hull of a set $\Pi_{i}$ of behavior strategies for player $i$ if there is an integer $k$, strategies $\left\{\pi_{i}^{j}\right\}_{j=1, k}$ in $\Pi_{i}$, sequences of

\footnotetext{
${ }^{5}$ Here and subsequently, we give the space of behavior strategies the norm topology, so that a sequence of behavior strategies converges iff it converges pointwise.
} 
strictly positive behavior strategies $\pi_{i}^{j, n} \rightarrow \pi_{i}^{j}$, and a sequence $\alpha^{n} \rightarrow \alpha$ of probability distributions on $[1, \ldots, k]$, such that the behavior strategies $\pi_{i}^{n}$ generated by the convex combination of $\pi_{i}^{1, n}, \pi_{i}^{2, n}, \ldots, \pi_{i}^{k, n}$ with weights $\alpha^{1, n}, \alpha^{2, n}, \ldots, \alpha^{k, n}$ converge to $\pi_{i \text {. }}$

We let $\alpha$ vary along the sequence so that the extensive-form convex hull will be closed. To see why the set would not be closed if the definition used only a fixed $\alpha$, consider the following one-player game. Player 1 has two moves in a row: The first choice is In or Out; Out ends the game, In gives him a second choice of L or R. Strategy 1 is (Out, L), 2 is (In, R). Now suppose that the definition of convex hull used only fixed weights, and let $\alpha, 1-\alpha$ be the weights on strategies 1 and 2 . Since only strategy 2 plays In and enables the move in the second period, the convex combination of the two strategies with strictly positive weights is $((\alpha$ Out, $(1-\alpha) \mathrm{In}), \mathrm{R})$, which approaches (Out, R) as $\alpha \rightarrow 1$. However, this is not a convex combination of strategies 1 and 2 , even for $\alpha=(1,0)$. Consequently, the set of "convex combinations" by this definition is not closed. Our definition of the extensive-form convex hull includes both (Out, L) and (Out, R).

Definition 2.2 (revised): A belief model $V$ is belief closed if for every $\left(\pi_{i},\left(a_{i}, \pi_{-i}^{i}\right)\right) \in V_{i}$, $\pi_{j}^{i}$ is in the extensive-form convex hull of the set $\left\{\pi_{j}^{\prime} \mid\left(\pi_{j}^{\prime}, b_{j}\right) \in V_{j}\right.$ for some belief $\left.b_{j}\right\}$.

All of the other definitions in our 1999 paper stay unchanged, modulo the change in the definition of belief closed. Note that the difference between the corrected definition of belief closed and the previous one arises when for some players $i$ and $j, \pi_{j}^{i}$ induces the same distribution over outcomes as a mixture over the set $\left\{\pi_{j}^{\prime} \mid\left(\pi_{j}^{\prime}, b_{j}\right) \in V_{j}\right.$ for some belief $\left.b_{j}\right\}$, but differs from these strategies at an information set that the strategies themselves preclude. For this reason, the changed definition of belief closed has only a minor effect on the concept of "rationalizability at reachable nodes" (definition 2.3), as this concept does not require that strategies be optimal at 
information sets that the strategies themselves rule out. In particular, while a given belief model $V$ (such as the pair of singleton beliefs $V_{1}, V_{2}$ in the example of the last section) can be rationalizable under reachable nodes under the old definition but not under the new one (because it is not belief-closed), for any such $V$ we can construct a $\hat{V}$ that is belief-closed by adding to each $V_{j}$ and every $\pi_{j} \in V_{j}$, every strategy $\hat{\pi}_{j}$ that agrees with $\pi_{j}$ at nodes that are reachable under $\pi_{j}$. Every added strategy is a best response at reachable nodes to the same beliefs that rationalized the original $\pi_{j}$, and since the set $V$ was belief closed under the old definition, $\hat{V}$ is belief closed under the new one. ${ }^{6}$ In particular, the change in definitions has no effect on whether a strategy profile $\hat{\pi}$ is a rationalizable self-confirming equilibrium, as this requires that there exist a belief model $V$ that is rationalizable at reachable nodes, such that for all players $i$, every $\left(\pi_{i}, b_{i}\right) \in V_{i}$ has the distribution of outcomes induced by $\hat{\pi}$. Thus Theorems 2.1, 4.1, and all of the examples in Section 3 are unaffected by the change.

As we noted earlier, the change in definition does matter for the concept of sequential rationalizability, which requires that strategies in the belief model be rationalized at every information set, and it has a similar impact on the concept of a sequentially rationalizable self-confirming equilibrium. In particular, because the extensive-form convex hull of a singleton set consists solely of the single strategy in that set, the unique sequentially rationalizable profile in finite games of perfect information with generic payoffs is the one given by backwards induction. ${ }^{7}$ Despite this change, theorem 4.2, which is the only result in [5] that refers to concepts using sequential

\footnotetext{
${ }^{6}$ Note that when a model $V$ that is rationalizable at reachable nodes under the old definition, the model formed by enlarging the set of strategies in each $V_{j}$ to its extensive-from convex hull need not be rationalizable at reachable nodes under the corrected definition. Although the new model will be beliefclosed, the strategies introduced need not be rational at reachable nodes, and indeed they may be strictly dominated.

${ }^{7}$ Bernheim [3] defines subgame rationalizability, and argues that it yields backwards induction in generic game of perfect information.
} 
rationality, is correct as stated, since the elaborations used in the proof have a type that is indifferent between all actions at every information set.

Note finally that that even if a strategy profile $\pi$ is sequentially rationalizable as a singleton set (i.e., there are beliefs $b_{i}$ for each player $i$ such that the sets $V_{1}=\left\{\left(\pi_{1}, b_{1}\right)\right\}$, $V_{2}=\left\{\left(\pi_{2}, b_{2}\right)\right\}$, etc. is sequentially rationalizable) it need not be a sequential equilibrium. While we have assumed that each player's assessment over nodes in his information sets is consistent in the Kreps-Wilson sense of being derivable from the limit of Bayesian beliefs from full-support strategies, we have not required that all players' assessments be consistent with a single sequence of totally mixed strategy profiles, and it is known, see for instance Example 8.5 in Fudenberg and Tirole [6], that the freedom to use different sequences to derive each player's assessment can allow additional equilibrium outcomes. 


\section{References}

1. G. Asheim and A. Perea, Lexicographic Probabilities and Rationalizability in Extensive-Form Games, mimeo, 2000.

2. P. Battigalli, Strategic Independence and Perfect Bayesian Equilibria, J. Econ. Theory 70 (1976), 201-234.

3. D. Bernheim, Rationalizable Strategic Behavior, Econometrica, 52 (1984), 10071028.

4. L. Blume, L., A. Brandenburger, and E. Dekel, Lexicographic Probabilities and Equilibrium Refinements, Econometrica 59 (1991), 81-98.

5. E. Dekel, D. Fudenberg, and D.K. Levine, Payoff Information and SelfConfirming Equilibrium, J. Econ. Theory 89 (1999), 165-185.

6. D. Fudenberg, and J. Tirole, "Game Theory," MIT Press, Cambridge, MA, 1991.

7. D. Kreps and R. Wilson, Sequential Equilibrium, Econometrica, 50 (1982), 863894

8. R. Myerson, Axiomatic Foundations of Bayesian Decision Theory, Center for Mathematical Studies in Economics and Management Science, Northwestern University Working Paper No. 671, 1986

9. D. Pearce, Rationalizable Strategic Behavior and the Problem of Perfection, Econometrica, 52 (1984), 1029-1050. 
Figure 1

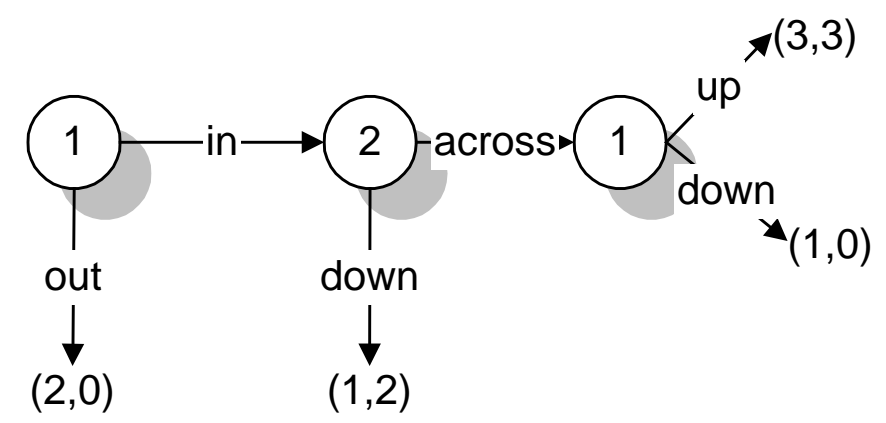

